\title{
Ovarian fibrothecoma
}

INSERM

\section{Source}

INSERM. (1999). Orphanet: an online rare disease and orphan drug data base. Ovarian fibrothecoma. ORPHA:314478

Ovarian fibrothecoma is a rare, benign, sex cord-stromal neoplasm, with a typically unilateral location in the ovary, characterized by mixed features of both fibroma and thecoma. Patients may be asymptomatic or may present with pelvic/abdominal pain and/or distension and, occasionally, with post-menopausal bleeding. Large tumors $(>10 \mathrm{~cm})$ are often associated with pleural effusion and ascites (the Meig's syndrome triad). 